\title{
Split Bregman iteration for hybrid regularization based image restoration
}

\author{
Weifeng Zhou ${ }^{a, b}$, Qingguo Li ${ }^{a *}$ and Lin Liang ${ }^{b}$ \\ ${ }^{a}$ College of Mathematics and Econometrics, Hunan University, Changsha, Hunan 410082, PR China \\ ${ }^{b}$ Department of Mathematics, Chuxiong Normal University, Chuxiong, Yunnan 675000, PR China
}

\begin{abstract}
In this paper, the hybrid regularization based scheme for image restoration is researched in detail. This strategy contains two regularization items, which helps to achieve superior image reconstruction. To quickly minimize the energy functional, we adopt the fast split Bregman iteration method. Numerical simulations distinctly demonstrate the superiority of our advanced scheme for image recovery over the traditional method.
\end{abstract}

Keywords. Image restoration; optimization problem; split Bregman iteration

\section{Introduction}

Image restoration is an inverse problem, which has been widely applied in various areas. Recently, partial differential equation based approaches for image recovery have made tremendous progress. The pioneer idea proposed by Tikhonov and Arsenin [1] can be characterized as follows

$$
\min _{u} \int_{\Omega}|\nabla u|^{2}+\frac{\lambda}{2} \int_{\Omega}|K u-f|^{2} \mathrm{~d} x
$$

where $u$ denotes the original image, $f$ is the observed degraded image, and $K$ stands for a bounded linear blur operator of $L^{2}(\Omega)$. They minimized this energy successfully when $u \in W^{1,2}(\Omega)$. Objectively, this scheme has the capability to remove noise well, but frequently causes edge blur.

To better recover and preserve the important edge details, Rudin, Osher, and Fatemi [2] proposed the following total variation (TV) regularization based image restoration algorithm (the ROF model)

$$
\min _{u} \int_{\Omega}|\nabla u|+\frac{\lambda}{2} \int_{\Omega}|K u-f|^{2} \mathrm{~d} x .
$$

This method maintains edges well while removing noise. For this reason, the classical ROF method has been studied by scholars at home and abroad, and emerged some fast numerical algorithms, such as fixed-point iteration method [3-7], dual method [8-9], augmented Lagrangian method [10-13], Bregman

${ }^{*}$ Corresponding author. Tel./fax: +86 73188822855 .

E-mail address: wfzhouqd@163.com (W. Zhou), liqingguoli@yahoo.com.cn (Q. Li). 
iteration [14-16], linearized Bregman iteration [17-21], and split Bregman iteration [16,22-25], alternating minimization and alternating direction algorithm [26,27], etc.

Unfortunately, the disgusting "staircase effect" inevitably arises in the homogeneous smooth region of the image caused by the ROF model. For better improving the quality of the recovered image, several authors investigated the hybrid regularization based schemes. For instance, total bounded variation regularization model [28], total variation and Besov regularizers [23], a hybrid of $H^{1}$ norm and TV norm regularizers [29-32]. Moreover, to reduce the staircasing effect, Tony et. al. [33] investigated the mixed regularization models for image decomposition and restoration.

Enlightened by the models mentioned above, we propose the following scheme:

$$
\min _{u} \int_{\Omega}|\nabla u|+\frac{\alpha}{2} \int_{\Omega}|\Delta u|^{2}+\frac{\beta}{2} \int_{\Omega}|K u-f|^{2} \mathrm{~d} x,
$$

where $\Omega$ is an open bounded domain with Lipschitzian boundary, $\alpha$ and $\beta$ denote the given positive parameters. An important remark is that, when $\alpha=0$, the model (1.3) can be changed into the classical ROF model (1.2). Complementally, for the proposed model (1.3), the TV regularization has the capability for preserving the edge details. The higher order derivative, located in the second term of (1.3), can be used for reducing the staircasing effect drastically.

The rest of this paper is arranged as follows. Section 2 focuses on the numerical algorithm: split Bregman iteration for solving the proposed scheme. Results of numerical experiments to indicate the better image reconstruction ability of our novel model, compared with the classical TV regularization based version, are shown in Section 3. Finally, the conclusion is given in Section 4.

\section{Numerical algorithm}

In this section, our objective is to employ the split Bregman iteration for minimizing the energy functional (1.3). Namely, we want to solve the following optimization problem

$$
u=\arg \min _{u}\|\nabla u\|_{1}+\frac{\alpha}{2} \int_{\Omega}|\Delta u|^{2}+\frac{\beta}{2} \int_{\Omega}|K u-f|^{2} \mathrm{~d} x .
$$

The split Bregman iteration initially introduced in image processing by Goldstein and Osher [23], can effectively solve this nondifferential problem. By introducing an auxiliary variable, they solved the differentiable $L^{2}$ norm problem rather than the non-differentiable $L^{1}$ norm one. To some extent, this manipulation can significantly increase the speed of numerical calculation.

Motivated by the ideas of [23], we firstly replace $\nabla u$ by $d$. This leads to the constrained problem as follows

$$
\min _{u, d}\|d\|_{1}+\frac{\alpha}{2} \int_{\Omega}|\Delta u|^{2}+\frac{\beta}{2} \int_{\Omega}|K u-f|^{2} \mathrm{~d} x, \text { such that } d=\nabla u .
$$

To make (2.2) easier to solve, we equivalently transform it into an unconstrained one as

$$
\min _{u, d}\|d\|_{1}+\frac{\alpha}{2} \int_{\Omega}|\Delta u|^{2}+\frac{\beta}{2} \int_{\Omega}|K u-f|^{2} \mathrm{~d} x+\frac{\gamma}{2}\|d-\nabla u\|_{2}^{2} .
$$


Then the elegant split Bregman iteration method solves (2.1) can be depicted as

$$
\begin{gathered}
\left(u^{k+1}, d^{k+1}\right)=\arg \min _{u, d}\|d\|_{1}+\frac{\alpha}{2} \int_{\Omega}|\Delta u|^{2}+\frac{\beta}{2} \int_{\Omega}|K u-f|^{2} \mathrm{~d} x+\frac{\gamma}{2}\left\|d-\nabla u-b^{k}\right\|_{2}^{2}, \\
b^{k+1}=b^{k}+\left(\nabla u^{k+1}-d^{k+1}\right) .
\end{gathered}
$$

Obviously, the system (2.4) consists two decoupled $u$ and $d$ subproblems. Given $u^{0}=0, d^{0}=b^{0}=0$ and fixed the inner iteration to be one, then we get the following alternating split Bregman method

$$
\left\{\begin{array}{l}
u^{k+1}=\arg \min _{u} \frac{\alpha}{2} \int_{\Omega}|\Delta u|^{2}+\frac{\beta}{2} \int_{\Omega}|K u-f|^{2} \mathrm{~d} x+\frac{\gamma}{2}\left\|\nabla u-d^{k}+b^{k}\right\|_{2}^{2}, \\
d^{k+1}=\arg \min _{d}\|d\|_{1}+\frac{\gamma}{2}\left\|d-\nabla u^{k+1}-b^{k}\right\|_{2}^{2}, \\
b^{k+1}=b^{k}+\left(\nabla u^{k+1}-d^{k+1}\right) .
\end{array}\right.
$$

The subproblem for $u$ can be easily solved by the fast Fourier transform method by the following formula:

$$
u^{k+1}=\left[\beta K^{T} K+\alpha \Delta^{2}-\gamma \Delta\right]^{-1}\left(\beta K^{T} f+\gamma \nabla^{T}\left(d^{k}-b^{k}\right)\right) .
$$

As for the $d$ subproblem, mentioned in the second equation of (2.6), we apply the soft shrinkage operator of Wavelet analysis to solve the numerical difficulties caused by the non-differentiable term.

As a conclusion, these manipulations lead to an alternating minimization algorithm for solving the proposed methodology (1.3), displayed in Algorithm 1.

\section{Algorithm 1: Split Bregman iteration for solving the model (1.3)}

Initialization: $u^{0}=0$, and $d^{0}=b^{0}=0$;

While $\left\|u^{k+1}-u^{k}\right\|_{2}>$ tol

Compute $u^{k+1}=\left[\beta K^{T} K+\alpha \Delta^{2}-\gamma \Delta\right]^{-1}\left(\beta K^{T} f+\gamma \nabla^{T}\left(d^{k}-b^{k}\right)\right)$,

Compute $d^{k+1}=\max \left(\left\|\nabla u^{k+1}+b^{k}\right\|_{2}-\frac{1}{\gamma}, 0\right) \frac{\nabla u^{k+1}+b^{k}}{\left\|\nabla u^{k+1}+b^{k}\right\|_{2}}$;

Update $b^{k+1}=b^{k}+\left(\nabla u^{k+1}-d^{k+1}\right)$.

end

\section{Numerical simulations}

This section is devoted to illustrate the feasibility of our proposed scheme. We have compared our proposed method against the TV regularization based version. In order to quantitatively evaluate the image quality, we employ the signal noise ratio (SNR) as

$$
\mathrm{SNR}=10 \cdot \log _{10}\left(\frac{\sum_{i, j}[u(i, j)-\bar{u}(i, j)]^{2}}{\sum_{i, j}[n(i, j)-\bar{n}(i, j)]^{2}}\right),
$$


where $\bar{u}$ and $\bar{n}$ stand for the means of the image $u$ and the noise $n$, respectively. Generally, a higher SNR value implies the better quality image.

In Figure 1, we display the recovered results by applying two different models for image denoising. Concretely, Figure 1(a) is a clean lenna image with size 256 by 256. Figure 1(b) ( $\mathrm{SNR}=12.19 \mathrm{~dB}$ ) represents its degraded version by adding zero mean Gaussian noise with noise intensity 0.05. Our result, Figure 1(d) $(\mathrm{SNR}=16.91 \mathrm{~dB})$, has a higher SNR value than that of the ROF model (Figure 1(c): $\mathrm{SNR}=16.79 \mathrm{~dB}$ ). Meanwhile, to intuitively show the superiority of our proposed scheme, their local enlarged images are displayed in Figure 2, respectively. From Figure 2, it is distinct to observe that our recovered image (Figure 2(d)) has less staircasing effect than that of the ROF method (Figure 2(c)). Both recovered results are obtained by iterating 13 times. Moreover, the parameters for computing the hybrid regularization model are set to $\alpha=0.005$, and $\beta=4$. These show that the better reconstruction capabilities of our proposed novel scheme.

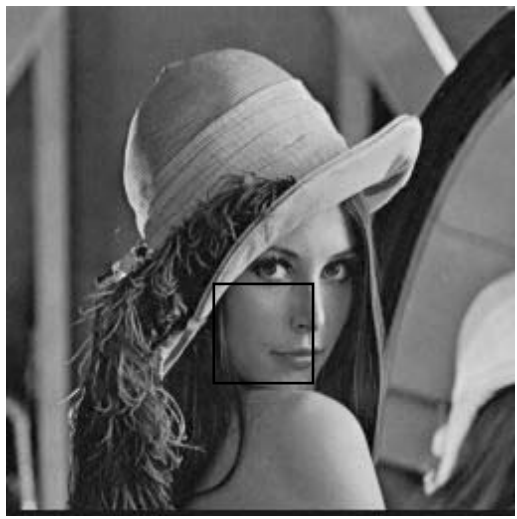

(a) Original image

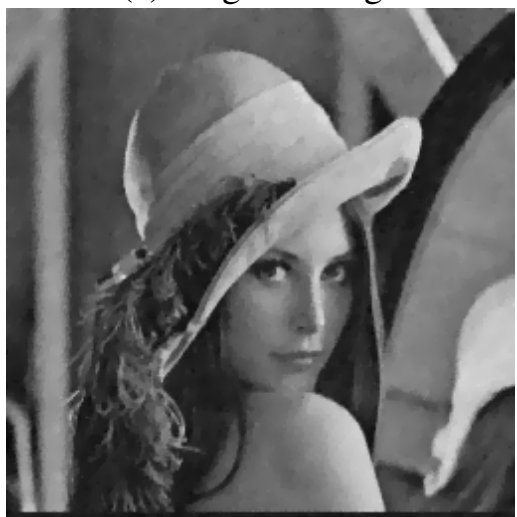

(c) The ROF model

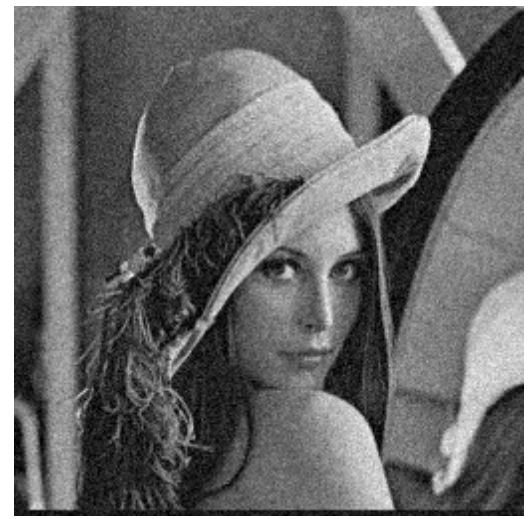

(b) Degraded image

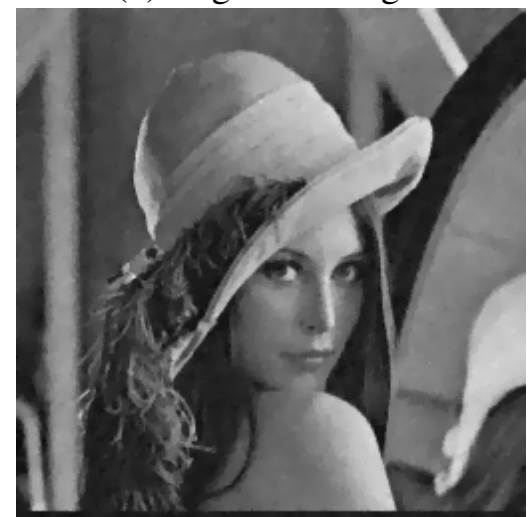

(d) Our proposed scheme

Figure 1: Recovered results via our proposed method and comparisons with that of the ROF model. 


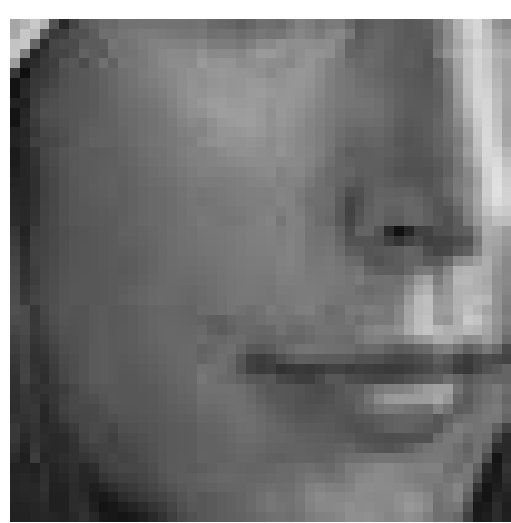

(a) Original image

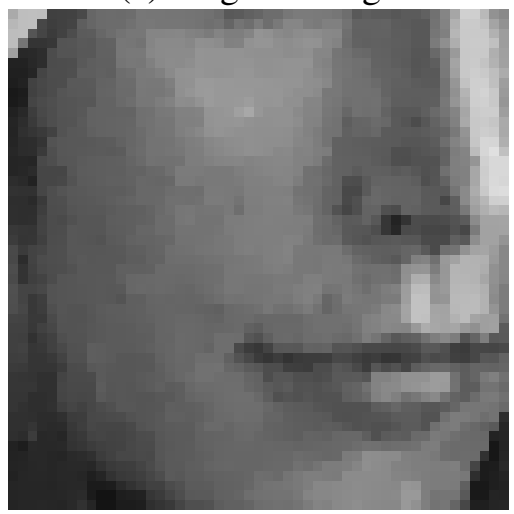

(c) The ROF model

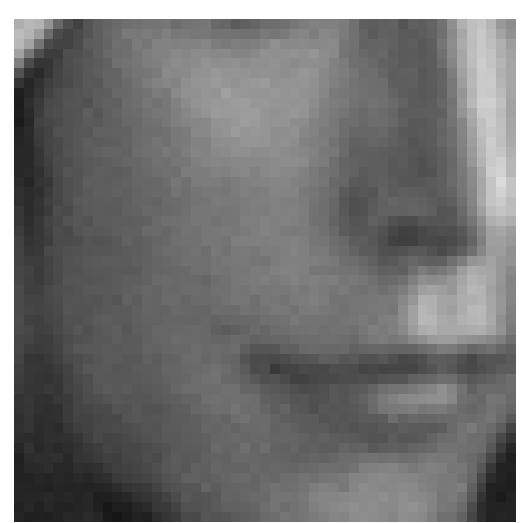

(b) Degraded image

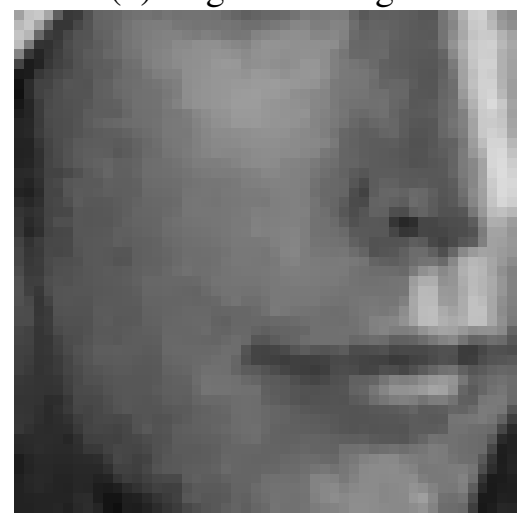

(d) Our proposed scheme

Figure 2: Recovered results via our proposed method and comparisons with that of the ROF model.

\section{Conclusion}

This article investigates the hybrid regularization based scheme for image restoration. To quickly minimize the energy functional, we propose the split Bregman iteration method. In comparison with the TV regularization based model, numerical results evidently demonstrate that our proposed strategy possesses the advantage in better improving the image reconstruction quality.

\section{Acknowledgments}

This work was supported by the National Natural Science Foundation of China (11071061) and the National Basic Research Program of China (2010CB334706).

\section{References}

[1] Tikhonov A.N., Arsenin V.Y. (1977). Solutions of Ill-Posed Problems, Winston and Sons, Washington, DC. 
[2] Rudin L., Osher S., Fatemi E. (1992). Nonlinear total variation based noise removal algorithms, Physica D, 60, 259-268.

[3] Acar R., Vogel C.R.(1994). Analysis of total variation penalty methods for ill-posed problems, Inverse Problems, 10, 1217-1229.

[4] Chambolle A., Lions P.L.(1997). Image recovery via total variational minimization and related problems, Numer. Math, 76, 167-188.

[5] Chan T.F., Mulet P.(1999). On the convergence of the lagged diffusivity fixed point method in total variation image restoration, SIAM J. Numer. Anal, 36, 354-367.

[6] Vogel C., Oman M. (1996). Iteration methods for total variation denoising, SIAM J. Sci. Comput, 17, 227-238.

[7] Dobson D., Vogel C.R. (1997). Convergence of an iterative method for total variation denoising, SIAM J. Numer. Anal, 34, 1779-1791.

[8] Chan T.F., Golub G.H., and Mulet P. (1999). A nonlinear primal-dual method for total variationbased image restoration, SIAM J. Sci. Comput, 20, 1964-1977.

[9] Chambolle A. (2004). An algorithm for total variation minimization and application, J. Math. Imaging Vis, 20, 89-97.

[10] Esser E. (2009). Applications of Lagrangian-based alternating direction methods and connections to split Bregman, UCLA, CAM Report 09-31.

[11] Wen Z., Goldfarb D., and Yin W. (2010). Alternating direction augmented Lagrangian methods for semidefinite programming, Math. Prog. Comp. 2, 203-230.

[12] Wu C., Tai X.-C. (2010). Augmented Lagrangian method, dual methods, and split Bregman iteration for ROF, vectorial TV, and high order models, SIAM J. Imaging Sci, 3, 300-339.

[13] Wu C., Zhang J., and Tai X.-C.. (2011). Augmented Lagrangian method for total variation restoration with non-quadratic fidelity, Inverse Probl. Imaging, 5(1), 237-261.

[14] Osher S., Burger M., Goldfarb D., Xu J., and Yin W. (2005). An iterative regularization method for total variation-based image restoration. Multiscale Model. Simul. 4, 460-489.

[15] Wang Y., Yang J., Yin W., and Zhang Y. (2008). A new alternating minimization algorithm for total variation image reconstruction. SIAM J. Imaging Sci, 1, 248-272.

[16] Yin W., Osher S., Goldfarb D., and Darbon J. (2008). Bregman iterative algorithms for $L^{1}$ minimization with applications to compressend sensing. SIAM J. Imaging Sci, 1, 143-168.

[17] Darbon J., Osher S., (2007). Fast discrete optimization for sparse approximations and deconvolutions, preprint. 
[18] Cai J.F., Osher S., Shen Z. (2009). Linearized Bregman iterations for compressed sensing, Math. Comp, 78, 1515-1536.

[19] Cai J.F., Osher S., Shen Z.W. (2009). Convergence of the linearized Bregman iteration for 11-norm minimization, Math. Comp, 78, 2127-2136.

[20] Osher S., Mao Y., Dong B., Yin W. (2008). Fast linearized Bregman iterations for compressed sensing and sparse denoising, UCLA, CAM Report 08-37.

[21] Yin W. (2010). Analysis and generalizations of the linearized Bregman method, SIAM J. Imaging Sciences, 3, 856-877.

[22] Cai J.F., Osher S., and Shen Z. (2010). Split Bregman methods and frame based image restoration, Multiscale Model. Simul, 8, 337-369.

[23] Goldstein T., Osher S. (2009). The Split Bregman algorithm for $L^{1}$ regularized problems. SIAM J. Imaging Sci, 2, 323-343.

[24] Wang Y., J. Yang Y., Yin W., Zhang Y. (2007). A new alternating minimization algorithm for total variation image reconstruction, CAAM Technical Report, TR07-10.

[25] Jia R.-Q., Zhao H., Zhao W. (2009). Convergence analysis of the Bregman method for the variational model of image denoising, Appl. Comput. Harmon. Anal, 27, 367-379.

[26] Zhou W., Li Q. (2013). Adaptive total variation regularization based Scheme for Poisson noise removal, Math Method Appl Sci, 36, 290-299.

[27] Zhou W., Li Q. (2012). Poisson Noise Removal Scheme Based on Fourth-Order PDE by Alternating Minimization Algorithm, ABSTR APPL ANAL, doi:10.1155/2012/965281.

[28] Hintermüller M., Kunisch K. (2004). Total bounded variation regularization as a bilaterally constrained optimization problem, SIAM J. Appl. Math, 64, 1311-1333.

[29] Ito K., Kunisch K. (1999). An active set strategy based on the augmented Lagrangian formulation for image restoration, Math. Model. Numer. Anal, 33, 1-21.

[30] Kärkkäinen T., Majava K. (2000). Nonmonotone and monotone active-set methods for image restoration, part I: convergence analysis, J. Optim. Theory Appl, 106, 61-80.

[31] Kärkkäinen T., Majava K. (2000). Nonmonotone and monotone active-set methods for image restoration, part II: numerical results, J. Optim. Theory Appl, 106, 81-105.

[32] Hintermüller M., Stadler G. (2006). An infeasible primal-dual algorithm for TV-based infconvolution-type image restoration, SIAM J. Sci. Comput, 28, 1-23.

[33] Chan T.F., Esedoglu S., Park F.E. (2007). Image decomposition combining staircase reduction and texture extraction, J. Vis. Commun. Image R, 18, 464-486. 